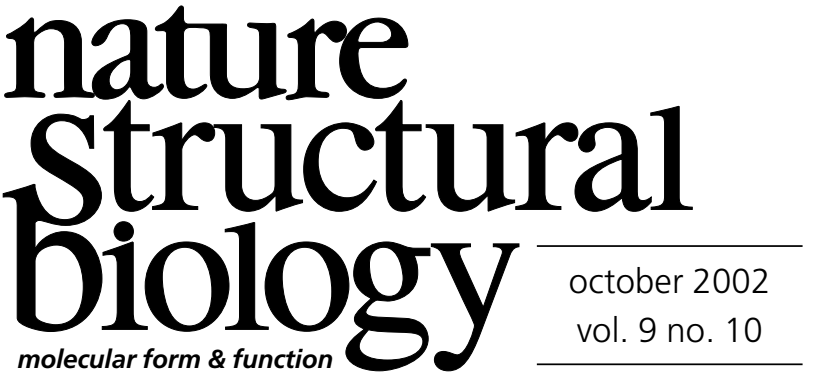

\section{Folding as grammar}

The following sentence is one that turns up frequently in a referee's report, especially when the report is on a revised version:

This manuscript is not publishable.

However, this sentence contains a typographical error that completely negates its intended meaning. In the language of molecular biology, this sentence contains a point mutation.

The analogy between language and molecular biology can be extended further than this somewhat superficial example might suggest. Considering a sentence, it consists of a linear sequence of 27 letters (including spaces); short local regions of the sentence can have an autonomous existence (we call these 'words') but the meaning of the sentence as a whole only emerges when all these disparate words are brought together, often modifying words far removed from them in sequence.

If that description sounds vaguely familiar, it should. Just change 'sentence' to 'protein', 'letters' to 'amino acids', 'words' to 'secondary structure elements' and 'meaning' to 'function', and you get: "Considering a protein, it consists of a linear sequence of 20 amino acids; short local regions of the protein can have an autonomous existence (we call these 'secondary structure elements') but the function of the protein as a whole only emerges when all these disparate secondary structure elements are brought together, often modifying secondary structure elements far removed from them in sequence." This implies that, at some level, sentences and proteins share an underlying logic.

Here is another example of sentences displaying a feature of protein structure; a domain swap from Lewis Carroll's Alice's Adventures in Wonderland:

"For the Duchess; an invitation from the Queen to play croquet"

"From the Queen; an invitation for the Duchess to play croquet"

Finding, or inventing, such analogs isn't just a parlor game. Many of the increasingly sophisticated bioinformatics programs used for identifying genes in genomic sequences employ Hidden Markov Models (HMM), a mathematical approach that emerged from the study of formal grammars and was first widely employed for automatic speech recognition (for reviews, see refs 1,2$)$. Such grammatical analysis has also been employed with considerable success in the prediction of RNA structures from their sequences (see ref. 3 ).

If bioinformatics can exploit the ideas of formal grammar that emerged from the work of Noam Chomsky and others in the 1950 s, then what, in biological terms, is equivalent to the grammatical analysis that is needed to make sense of the string of words forming a sentence? For RNA and proteins the answer is simple: folding. Then our attempts to predict the structures of proteins and understand how they reach this final form becomes a quest to learn the grammar inherent in protein 'language'.

Anyone in need of convincing that this is an important goal need look no further than the new Horizon Symposium series, jointly created by the Nature Publishing Group and Aventis, the topic of whose first meeting is "Protein Folding and Disease". These symposia will address timely topics of relevance to the drug discovery and development community, but will focus mainly on the basic science of their subjects.

Horizon Symposia will bring together an interdisciplinary mix of scientists and give them more time devoted to discussion than is usual at most meetings. Although attendance at the meetings will be by invitation only, the Horizon Symposia website (http://www.nature.com/drugdisc/horizon/) will present an introduction to the topic of each symposium, as well as summaries of the findings and highlights of the debates, so that everybody with an interest in the subject can benefit from the meeting.

Much time at the first symposium will doubtless be spent discussing the particular properties of the proteins underlying amyloidoses and other folding diseases. Perhaps it will be useful to ponder whether some error in the grammar of their sequences lies behind their disease-causing behaviors. Are they correctly construed but in fact meaningless such as this sentence from Chomsky"

\section{Colourless green ideas sleep furiously.}

or contain some internal contradiction such as the exhortation:

\section{Moderation in all things, including moderation!}

Alternatively it may be most profitable to view prion proteins as biological sentences whose meaning/structure is ambiguous, only being resolved by their context/environment like this World War II newspaper headline:

Eighth army push bottles up Germans.

Finally, an explanation of the referee's report with which we began. The typo is that 'not' should read 'now', showing that point mutations can be as fatal for scientific papers as for the proteins and RNA, which are their subjects.

1. Searls, D.B. Bioinformatics 17, 579-80 (2001).

2. Yandell, M.D. \& Majoros, W.H. Nature Rev. Genet. 3, 601-610 (2002).

3. Rivas, E. \& Eddy, S.R. Bioinformatics 16, 334-40 (2000).

4. Chomsky, N. Syntactic Structures (Mouton, The Hague; 1957) 